\title{
Release Adiabat Measurements on Minerals: The Effect of Viscosity
}

\author{
Raymond Jeanloz' and Thomas J. Ahrens
}

\author{
Seismological Laboratory, Division of Geological and Planetary Sciences, California Institute of Technology \\ Pasadena, Califomia 91125
}

\begin{abstract}
The current inversion of pressure-particle velocity data for release from a high-pressure shock state to a pressure-density path usually depends critically upon the assumption that the release process is isentropic. It has been shown by Kieffer and Delaney that for geological materials below stresses of $\sim 150$ $\mathrm{GPa}$, the effective viscosity must be $\leqslant 10^{3} \mathrm{~kg} \mathrm{~m}^{-1} \mathrm{~s}^{-1}\left(10^{4} \mathrm{P}\right)$ in order that the viscous (irreversible) work carried out on the material in the shock state remains small in comparison to the mechanical work recovered upon adiabatic rarefaction. The available data pertaining to the offset of the Rayleigh line from the Hugoniot curve for minerals, the magnitude of the shear stress in the high-pressure shock state for minerals, and the direct measurements of the viscosities of several engineering materials shocked to pressures below, $150 \mathrm{GPa}$ yield effective viscosities of $\sim 10^{3} \mathrm{~kg} \mathrm{~m}^{-1} \mathrm{~s}^{-1}$ or less. We infer that this indicates that the conditions for isentropic release of minerals from shock states are achieved, at least approximately, and we conclude that the application of the Riemann integral to obtain pressure-density states along the release adiabats of minerals in shock experiments is valid.
\end{abstract}

Over the last decade an increasing number of measurements of dynamic unloading from high-pressure shock (Hugoniot) states have been reported for rocks and minerals, including porous samples and soils [Ahrens et al., 1969; Petersen et al., 1970; Lysne, 1970; Grady et al., 1974, 1975; Ahrens, 1975; Grady and Murri, 1976; Jeanloz and Ahrens, 1977, 1978; Jackson and Ahrens, 1979]. The release path measurements have been motivated by the information provided, which is complementary to that given by Hugoniot data in constraining the high-pressure equation of state as well as the nature of yielding and apparent phase transformations under shock conditions. Upon unloading, variables such as particle velocity or pressure in the sample are measured either discretely or continuously in time [Ahrens et al., 1969; Cowperthwaite and Williams, 1971; Grady, 1973; Seaman, 1974]. These are usually converted to release paths in the pressure-density plane by way of the Riemann integral formulation, which, however, is strictly valid only for isentropic (or isothermal) flows [e.g., Rice et al., 1958; Fowles and Williams, 1970; Lyzenga and Ahrens, 1978].

Recently, Kieffer and Delaney [1979] have demonstrated how the nondimensionalized expressions of Thompson [1972] for the conservation equations of a compressible, viscous fluid may be utilized to obtain criteria to determine whether the release processes from high-pressure shock states in materials of geophysical interest are indeed isentropic. The objects of the present note are both to clarify the derivation of these isentropic flow criteria and to point out how existing data, quite surprisingly, demonstrate that the effective viscosity of solids shocked to high pressures is sufficiently low that dynamic unloading appears to be isentropic.

Isentropic flow is assumed in the Riemann integral formulation because the change in pressure $\boldsymbol{P}$ with density $\rho$ is assumed to be given by the square of the isentropic bulk sound speed, $c^{2} \equiv(\partial P / \partial \rho)_{s}$. Hence the appropriate criterion for isentropic flow upon decompression is that the change in pressure associated with a change in entropy is negligibly small in comparison to the isentropic change in pressure. Since the change

\footnotetext{
' Present address: Department of Geological Sciences, Harvard University, Cambridge, Massachusetts 02138 .
}

Copyright $(\mathcal{C} 1979$ by the American Geophysical Union. in pressure in a volume element within the flow is given by

$$
\frac{D P}{D t}=c^{2} \frac{D \rho}{D t}+\left(\frac{\partial P}{\partial s}\right)_{\rho} \frac{D s}{D t}
$$

where $t$ is time, $s$ is specific entropy, and $D$ denotes Lagrangian differentiation, the criterion for isentropic flow is

$$
\left|c^{2} \frac{D \rho}{D t}\right| \gg\left|\left(\frac{\partial P}{\partial s}\right)_{\rho} \frac{D s}{D t}\right|
$$

However, $\nu \rho / D t=-\rho \nabla \cdot u$ from conservation of mass, while the right-hand side of (2) can be expanded by way of the following thermodynamic identity:

$$
\left(\frac{\partial P}{\partial s}\right)_{\rho}=-\left(\frac{\partial \rho}{\partial T}\right)_{P}\left(\frac{\partial T}{\partial s}\right)_{P} c^{2}
$$

Hence (2) becomes

$$
|\nabla \cdot \mathbf{u}| \gg\left|\frac{1}{\rho}\left(\frac{\partial \rho}{\partial T}\right)_{P}\left(\frac{\partial T}{\partial s}\right)_{P} \frac{D s}{D t}\right|
$$

where $c^{2}$ has been cancelled from both sides, $u$ is the particle velocity vector field, and $T$ is temperature. We consider two sources of entropy production, namely, those associated with mechanical dissipation and thermal dissipation [cf. Thompson, 1972, equation 2.1 b]:

$$
\rho \frac{D s}{D t}=\frac{\Gamma}{T}-\frac{\nabla \cdot q}{T}
$$

Here $\Gamma$ is the viscous dissipation function (units of energy/ time), and $q$ is the heat flux vector. In principle, both bulk and shear viscosities enter into $\Gamma$ (along with terms for plastic work); however, we will not differentiate between these but rather use an effective viscosity $\eta$ [see Gilman, 1979]. We note that (4) does not account for any reactions (including phase transformations) which might occur in the flow, such processes requiring an extra set of terms involving the affinities of the components [e.g., DeGroot and Mazur, 1969]. Also, this equation does not apply to a discontinuous production of entropy, such as occurs across the shock front, except in a limiting sense [Courant and Friedrichs, 1948, sect. 63]. Substituting (4) into (2') and using the heat conduction equation ( $\alpha$ is the thermal diffusivity, which is assumed constant), we get 


$$
|\nabla \cdot \mathbf{u}| \gg\left|\frac{1}{\rho^{2}}\left(\frac{\partial \rho}{\partial T}\right)_{P}\left(\frac{\partial T}{\partial s}\right)_{P}\left[\frac{\Gamma}{T}+\rho C_{P} \frac{\kappa}{T} \nabla^{2} T\right]\right|
$$

For one-dimensional flow and nonnegligible compression this reduces to

$$
|\nabla \cdot \mathrm{u}| \sim\left|u_{, x}\right| \gg\left|\left(\frac{\partial V}{\partial T}\right)_{P} \frac{T}{C_{P}}\left[\frac{\Gamma}{T}+\rho C_{P} \frac{\kappa}{T} T_{x, x}\right]\right|
$$

where subscript,$x$ indicates differentiation in the $x$ direction and $V$ and $C_{P}$ are the specific volume and specific heat at constant pressure, respectively.

Following Thompson [1972, p. 140], (5') can be normalized in terms of a characteristic length $l$, particle velocity $u_{0}$, volume $V_{0}$, and temperature $T_{0}$. With $X=x / l_{0}$ and $\Gamma_{0}=\eta u_{0}{ }^{2} / l_{0}{ }^{2}$, this becomes

$$
\left|\frac{u_{0}}{l_{0}}\left(\frac{u}{u_{0}}\right)_{x}\right| \gg\left|\left(\frac{\partial V}{\partial T}\right)_{P} \frac{T}{C_{P}}\left\{\frac{\eta u_{0}^{2}}{l_{0}^{2} T}\left(\frac{\Gamma}{\Gamma_{0}}\right)+\frac{\kappa}{T} \rho C_{P} \frac{T_{0}}{l_{0}^{2}}\left(\frac{T}{T_{0}}\right)_{, x, x}\right\}\right|
$$

and rearranging terms yields

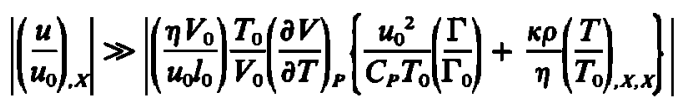

Because the normalized variables $\left(u / u_{0}\right)_{x},\left(\Gamma / \Gamma_{0}\right)$, and $(T /$ $\left.T_{0}\right)_{, x, x}$ have values near unity [see Thompson, 1972; Kieffer and Delaney, 1979], (7) may be rewritten as

$$
1 \gg\left|\frac{1}{R e_{0}} T_{0} \alpha \cdot\left\{\frac{u_{0}^{2}}{C_{P} T_{0}}+\frac{1}{\operatorname{Pr}}\right\}\right|
$$

where $u_{0} l_{0} / V_{0} \eta$ is the Reynolds number $R e_{0}, \alpha$ is the coefficient of thermal expansion, and $\operatorname{Pr}=\eta / \kappa \rho$ is the Prandtl num. ber. As Kieffer and Delaney [1979] pointed out, at temperatures of geophysical interest, $\alpha T_{0} \simeq 0.1$ for shocked minerals and liquids, and the inequality of (8) reduces to the two inequalities

$$
\begin{gathered}
\left|\frac{\kappa}{u_{0} l_{0}}\right| \ll 10 \\
\left|\frac{\eta}{\rho_{0} u_{0} l_{0}} \frac{u_{0}^{2}}{C_{P} T_{0}}\right| \ll 10
\end{gathered}
$$

which are identical to their equations $17 a$ and $17 b$. For shock waves in rocks and minerals the density is of the order of 5 $\mathrm{Mg} \mathrm{m}^{-3}$, characteristic particle velocities are of the order of $10^{3} \mathrm{~m} \mathrm{~s}^{-1}$, and $C_{P}$ and $\kappa$ are very nearly $10^{3} \mathrm{~J} \mathrm{~kg}^{-1} \mathrm{~K}^{-1}$ and

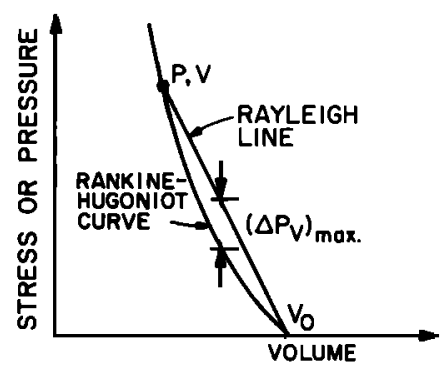

Fig. 1. Relation of Hugoniot state $(\boldsymbol{\mu}, \eta)$ and thermodynamic path (Rayleigh line) to Rankine-Hugoniot curve. The pressure difference $\left(\Delta P_{V}\right)_{\max }$ is considered a measure of the effective viscosity at the shock front.

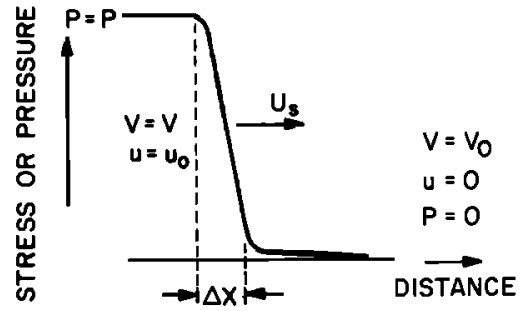

Fig. 2. Shock wave profile with finite width $\Delta X$ resulting from intrinsic material viscosity $\eta$ at the shock front.

$10^{-6} \mathrm{~m}^{2} \mathrm{~s}^{-1}$, respectively. The rise times of shock fronts provide a lower bound for $l_{0}$, and hence conservative bounds for the inequalities, of about $10^{-5} \mathrm{~m}$ [Grady, 1977]. The thermal inequality given by $(9 a)$ is easily satisfied, since the left-hand side is of magnitude $\sim 10^{-4}$. Hence heat flow contributes negligibly to entropy production, and release from the Hugoniot state is clearly adiabatic, as is commonly assumed.

On the other hand, the left-hand side of $(9 b)$ requires an effective viscosity $\eta \leq 10^{3} \mathrm{~kg} \mathrm{~m}^{-1} \mathrm{~s}^{-1}\left(10^{4} \mathrm{P}\right)$ for flow behind a shock wave to be isentropic $\left(T_{0}\right.$ is of order $10^{3} \mathrm{~K}$; Kieffer and Delaney [1979]). What then is the viscosity in the material behind a shock front in a solid? Duvall [1962] suggested, in a rather simple argument, that the thickness of a stable transition zone from the unshocked to the shocked state $\Delta X$ is related to the difference at constant volume between the Rayleigh line and a static (equilibrium) curve lyng wear the Hugoniot, $\Delta P_{V}$ (see Figures 1 and 2). Thus $\Delta X$ is determined by the effective viscosity $\eta$ times the ratio of the pressure jump $P$ to the maximum pressure gradient along the front (given by the maximum value of $\rho_{0} U_{S} \Delta P_{\nu}$, where $U_{S}$ is the shock wave velocity):

$$
\Delta X=\frac{\eta P}{\left.\rho_{0} U_{s} \Delta P_{V}\right)_{\max }}=\eta \frac{u_{0}}{\left.\Delta P_{V}\right)_{\max }}
$$

This means that as the viscosity increases, the rise time of the shock increases, as is expected. For shock fronts in silicates, $\Delta X \simeq 10^{-4} \mathrm{~m}$, while $\Delta P_{V} / P$ is of order $10^{-1}$ (or less) in the 10 o 100-GPa range. Appropriate values of $\rho_{0} U_{S}$ are $\sim 10^{7} \mathrm{~kg}$ $\mathrm{m}^{-2} \mathrm{~s}^{-1}$, and therefore $\eta \simeq 10^{3} \mathrm{~kg} \mathrm{~m}^{-1} \mathrm{~s}^{-1}$ is a conservative estimate from (10).

We note that this approach is analogous to the simple dimensional argument that the effective viscosity is given by the ratio of the deviatoric stress $\sigma$ to the strain rate $\dot{\varepsilon}$. Approximating the strain rate by

$$
\dot{\varepsilon} \sim \frac{1}{\tau} \frac{\Delta V}{V}=\frac{U_{s}}{\Delta X} \frac{\Delta V}{V}
$$

where $\tau$ is the rise time of the shock and $\Delta V / V$ is the compression, we arrive at a value of $\dot{\varepsilon} \sim 10^{8} \mathrm{~s}^{-1}$. In virtually all silicates and in many oxides the stress difference between the Hugoniot and the hydrostatic compression curve appears to be quite small at pressures in the 10- to 100-GPa range. For example, in the case of quartz this difference amounts to no more than $100 \mathrm{MPa}$ at $10 \mathrm{GPa}$ [Ahrens and Linde, 1968], while Ahrens et al. [1968] found a stress difference as large as about $3 \mathrm{GPa}$ in polycrystalline corundum. Identifying these stress differences with $\sigma$, we arrive at effective viscosities $\eta \sim$ $\sigma / \dot{\varepsilon}<10^{2} \mathrm{~kg} \mathrm{~m}^{-1} \mathrm{~s}^{-1}$. This conclusion is corroborated by recent rise- time measurements in metals using velocity-interferometer techniques [Chhabildas and Asay, 1979]. We there- 
fore conclude, on the basis of arguments such as those presented by Duvall [1962], that the effective viscosities of silicates and oxides appear to be low enough under shock conditions to satisfy the criteria for isentropic flow during release. It is also worth noting that the nature of the shock front is likely to reflect the properties of the material at high pressure behind the front. For a steady shock, any perturbation behind the front is communicated forward toward the shock front and overtakes the shock front, in the laboratory reference frame, at a speed $u+c$, where $c$ is the local sound speed. Since $u+c$ $>U_{s}$, the shock and particle velocities reflect the properties of the material encompassed by the shock [Duvall, 1978], and estimates of the viscosity from the rise time of the front should approximately correspond to the effective viscosity in the shocked state [cf. Chhabildas and Asay, 1979].

Probably the most conclusive evidence that the effective viscosity behind shock fronts in such solids as $\mathrm{Al}, \mathrm{Pb}, \mathrm{Fe}$, and $\mathrm{NaCl}$ is low, in the range $10^{3}-10^{4} \mathrm{~kg} \mathrm{~m}^{-1} \mathrm{~s}^{-1}$, is the experiments of Sakharov et al. [1965] and more recently Mineev and Savinov [1967] at shock pressures ranging from $\sim 12$ to 250 $\mathrm{GPa}$. In these experiments the decay of perturbations to the shock front induced by impact of an explosively driven, sinusoidally corrugated piston into the sample material has demonstrated that virtually all of these materials have similar effective viscosities. Moreover, experiments carried out by independent techniques on mercury and water [Mineev and Zaidel, 1968; Al'tshuler et al., 1977] demonstrate that these substances have comparable viscosities, $\sim 10^{3} \mathrm{~kg} \mathrm{~m}^{-1} \mathrm{~s}^{-1}$, in the 4- to 44-GPa range. Also, studies of jet formation during oblique impact of several metals by Godunov et al. [1971, 1974,1975 ] indicate similarly low viscosities at strain rates achieved upon shock loading. These results independently suggest that silicates may likewise have low, fluidlike viscosities under shock.

Finally, we note that the sound speed measurements of Grady et al. [1975] indicate velocities in quartz shocked to 22$35 \mathrm{GPa}$ which are well below the longitudinal velocity but consistent with velocities expected for a fluidlike mixture of quartz and stishovite. Similar results were obtained for feldspar shocked to pressures of $46 \mathrm{GPa}$, and Grady et al. [1975] infer from these anomalously low sound speeds that these minerals contain very hot and probably liquid zones (see also Grady [1977]). This again suggests that the effective viscosity of silicates under shock conditions may be quite low. We note, however, that alternative interpretations can be found to explain the reduced sound speeds. For example, they may be ascribed to a modulus defect associated with dispersion and due to an unknown absorption mechanism [e.g., Nowick and Berry, 1972; Thompson, 1972]; this would actually imply increased viscosities. Phase tranformations are not involved in reducing the sound speeds, however, since both corundum and periclase exhibit such reduced velocities under shock while undergoing no phase changes [Bless and Ahrens, 1976; Grady, 1977].

We conclude, on the basis of several lines of evidence, that the effective viscosity in shocked oxides and silicates is probably very low in comparison to unshocked materials, perhaps as low as $10^{3} \mathrm{~kg} \mathrm{~m}^{-1} \mathrm{~s}^{-1}$ or less. Such low values may represent a purely transient effect or may be the result of massive dynamic yielding, among other processes [e.g.; Gilman, 1979]. Godunov et al. [1974] present a simple phenomenological model which yields the observed low viscosities at the high strain rates achieved in shock. Although direct measurements of the viscosity of silicates and oxides in the shocked state have yet to be carried out, we conclude that the viscous inequality (equation $(9 a)$ ) is satisfied and that the rarefaction process described by release adiabats from the shock state is nearly isentropic (see also Chhabildas and Asay [1979]). The major assumptions in our analysis arise from our neglecting the effects of dissipation due to reactions and our not distinguishing between shear and other forms of mechanical dissipation; however, our result justifies the use of the Riemann integral formulation, which converts pressure-particle velocity states to pressure-density states along the release adiabat [Rice et al., 1958; Ahrens et al., 1969; Grady, 1977; Lyzenga and Ahrens, 1978]. Thus the additional data inferred from such release adiabat measurements provide valid, and in most cases, useful constraints on the equations of state of minerals.

Acknowledgments. We appreciate having received a preprint of the Kieffer and Delany paper and are particularly indebted to $D$. E. Grady, D. L. Anderson, S. W. Kieffer, and a reviewer for their helpful comments. This work was supported by the National Aeronautics and Space Administration under grant NGL-05-002-105. Contribution 3226, Division of Geological and Planetary Sciences, California Institute of Technology, Pasadena, California 91125.

\section{REFERENCES}

Ahrens, T. J., Compaction by impact of unconsolidated lunar fines, Moon, 14, 291-299, 1975.

Ahrens, T. J., and R. K. Linde, Response of brittle solids to shock compression, in Behavior of Dense Media Under High Dynamic Pressures, edited by J. Berger, pp. 325-336, Gordon and Breach, New York, 1968

Ahrens, T. J., W. H. Gust, and E. B. Royce, Material strength effect on the shock compression of alumina, J. Appl. Phys., 39, 4610-4616, 1968.

Ahrens, T. J., C. F. Peterson, and J. T. Rosenberg, Shock compression of feldspars, J. Geophys. Res., 74, 2727-2746, 1969.

Al'tshuler, L. V., G. I. Kanel', and B. S. Chekin, New measurements of the viscosity of water behind a shock wave front, Sov. Phys. JETP, 45, 348-350, 1977.

Bless, S. J., and T. J. Ahrens, Measurement of release wave speed in shock-compressed polycrystalline alumina and aluminum, $J$. Geophys. Res., 81, 1935-1942, 1976.

Chhabildas, L. C., and J. R. Asay, Rise-time measurements of shock transitions in aluminum, copper, and steel, J. Appl. Phys., 50, 27492756, 1979.

Cowperthwaite, M., and R. F. Williams, Determination of constitutive relationships with multiple gauges in nondivergent waves, $J$. Appl. Phys., 42, 456-462, 1971.

Courant, R., and K. O. Fredrichs, Supersonic Flow and Shock Waves, 464 pp., Springer, New York, 1948.

DeGroot, S. R., and P. Mazur, Non-Equilibrium Thermodynamics, 510 pp., North-Holland, Amsterdam, 1969.

Duvall, G. E., Concepts of shock wave propagation, Bull. Seismol. Soc. Amer., 52, 869-893, 1962.

Duvall, G. E., Maxwell-like relations in condensed materials: Decay of shock waves, Iranian J. Sci. Technol., 7, 57-69, 1978.

Fowles, R., and R. F. Williams, Plane stress wave propagation in solids, J. Appl. Phys., 41, 360-363, 1970.

Gilman, J. J., Resistance to shock-front propagation in solids, J. Appl. Phys., 50, 4059-4064, 1979.

Godunov, S. K., A. A. Deribas, I. D. Zakharenko, and V. I. Mali, Investigation of the viscosity of metals in high-velocity collisions, Combust. Explos. Shock Waves USSR, Engl. Transl., 7, 114-118, 1971.

Godunov, S. K., A. F. Demchuk, N. S. Kozin, and V. I. Mali, Interpolation formulas for Maxwell viscosity of certain metals as a function of shear-strain intensity and temperature, J. Appl. Mech. Tech. Phys. USSR, Engl. Transl., 15, 526-529, 1974.

Godunov, S. K., A. A. Deribas, and V. I. Mali, Influence of material viscosity on the jet formation process during collisions of metal plates, Combust. Explos. Shock Waves USSR, Engl. Transl., II, 113, 1975 . 
Grady, D. E., Experimental analysis of alternating wave propagation, J. Geophys. Res., 78, 1299-1308, 1973.

Grady, D. E., Processes occurring in shock-wave compression of rocks and minerals, in High-Pressure Research: Applications to Geophysics, edited by M. H. Manghnani and S. Akimoto, pp. 389-438, Academic, New York, 1977.

Grady, D. E., and W. J. Murri, Dynamic unloading in shock compressed feldspar, Geophys. Res. Lett., 3, 472-474, 1976.

Grady, D. E., W. J. Murri, and G. R. Fowles, Quartz to stishovite: Wave propagation in the mixed phase region, J. Geophys. Res., 79 332-338, 1974.

Grady, D. E., W. J. Murri, and P. S. DeCarli, Hugoniot sound velocities and phase transformation in two silicates, J. Geophys. Res., 80, 4857-4861, 1975.

Jackson, I., and T. J. Ahrens, Shock wave compression of single crystal forsterite, J. Geophys. Res., 84, 3039-3048, 1979.

Jeanloz, R., and T. J. Ahrens, Pyroxenes and olivines: Structural implications of shock-wave data for high pressure phases, in HighPressure Research: Applications to Geophysics, edited by M. Manghnani and S. Akimoto, pp. 439-462, Academic, New York, 1977.

Jeanloz, R., and T. J. Ahrens, The equation of state of a lunar anorthosite: 60025, Proc. Lunar Planet. Sci. Conf. 9th, 2789-2803, 1978.

Kieffer, S. W., and J. M. Delany, Isentropic decompression of fluids from crustal and mantle pressures, $J$. Geophys. Res., 84, 1611-1620, 1979.

Lysne, P. C., A comparison of calculated and measured low-stress Hugoniots and release adiabats of dry and water-saturated tuff, $J$. Geophys. Res., 75, 4375-4386, 1970.
Lyzenga, G., and T. J. Ahrens, The relations between the shock-induced free-surface velocity and post-shock density of solids, $J$. Appl. Phys., 49, 201-204, 1978.

Mineev, V. N., and E. V. Savinov, Viscosity and melting point of aluminum, lead and sodium chloride subjected to shock compression, Sov. Phys. JETP, 25, 41 1-415, 1967.

Mineev, V. N., and R. M. Zaidel, The viscosity of water and mercury under shock loading, Sov. Phys. JETP, 27, 874-878, 1968.

Nowick, A. S., and B. S. Berry, Anelastic Relaxation in Crystalline Solids, 677 pp., Academic, New York, 1972.

Petersen, C. F., W. T. Murri, and M. Cowperthwaite, Hugoniot and release-adiabat measurements for selected geological materials, $J$. Geophys. Res., 75, 2063-2072, 1970.

Rice, M. H., R. G. McQueen, and J. M. Walsh, Compression of solids by strong shock waves, Solid State Phys. 6, 1-63, 1958.

Sakharov, A. D., R. M. Zaidel', V. N. Mineev, and A. G. Oleinik, Experimental investigations of the stability of shock waves and the mechanical properties of substances at high pressures and temperatures, Sov. Phys. Dokl., 9l, 1091-1094, 1965.

Seaman, $\mathbf{L}$., Lagrangian analysis for multiple stress or velocity gages in attenuating waves, J. Appl. Phys., 45, 4303-4314, 1974.

Thompson, P. A., Compressible Fluid Dynamics, 665 pp., McGrawHill, New York, 1972.

(Received June 8, 1979; accepted August 27, 1979. ) 